\title{
Simulations of Grant Allocation in NG-PON2 Networks Using OPNET Modeler
}

\author{
Tomas Horvath, Member, IEEE, Patrik Cymorek, Petr Munster, Member, IEEE, Vaclav Oujezsky, \\ and Josef Vojtech, Senior member, IEEE
}

\begin{abstract}
PONs (Passive Optical Networks) are the most promising networks for the future. In 1998, the first standard of PONs was developed. Since that time, quality of optical transmissions and technologies has been massively improved and today's standards are capable of transferring an enormous amount of data. For example, NG-PON2 networks are able to transfer up to $40 \mathrm{Gbit} / \mathrm{s}$, which means these networks provide subscribers with a symmetric bandwidth up to $10 \mathrm{Gbit} / \mathrm{s}$ using 4 different wavelengths. The higher transmission speeds are required, the more sophisticated bandwidth allocation reflecting requirements of each ONU (Optical Network Unit) in real-time is necessary. This article deals with implementation of NG-PON2 networks using OPNET Modeler and simulations of static and dynamic grants allocations. We also present a modification of dynamic bandwidth allocation for increasing the free bandwidth of idle ONU to heavily loaded ONU(s). We propose a simplified NG-PON2 (Next-Generation PON stage 2) network, for example, activation machine for ONUs is not implemented. At present, OPNET Modeler is being used to simulate EPON (Ethernet PON) networks that use a different approach for data transmission. Thanks to its own implementation, basic data transmission in NG-PON2 networks, and simulations of algorithms for dynamic bandwidth allocation can be achieved.
\end{abstract}

Index Terms-NG-PON2; OPNET Modeler; static grant allocation; dynamic grant allocation.

\section{INTRODUCTION}

During the last decade(s), the amount of internet customers massively increased. In consequence, demands for high-speed internet connection allowing a comfortable usage of modern technologies such as streaming, online gaming, HD IPTV (High Definition Internet Television Protocol), Netflix etc. increased as well. To satisfy the needs of today's users, PON networks are being deployed in urban and strongly inhabited areas.

ITU-T (International Telecommunication Union Telecommunication Standardization Sector) standards of PONs as GPON (Gigabit PON) [1], XG-PON (10 Gigabit PON) [2] and NG-PON2 (Next-Generation Passive Optical Network) [3] are focused on connecting many subscribers to network using P2MP (Point-to-Multipoint) structure of communication. In this structure of networks, TDM (Time

Manuscript received March 30, 2018; revised July 09, 2018. Date of publication October 03, 2018. The associate editor Prof. Ivan Radoš has been coordinating the review of this manuscript and approved it for publication.

Authors are with the Department of Telecommunication, Brno University of Technology, Technicka 12, Czech Republic. E-mails: (horvath, cymorek, munster, oujezsky, vojtech)@feec.vutbr.cz

Digital Object Identifier (DOI): 10.24138/jcomss.v14i4.525
Division Multiplex) and TDMA (Time Division Multiplex Access) are used. The aforementioned networks are deployed also in P2P (Point-to-Point) structure, but in most cases, P2MP structured networks are used.

Latest NG-PON2 (Next-Generation Passive Optical Network 2) standard is the first standard combining multiple wavelengths in downstream direction. Thanks to a WDM (Wavelength Division Multiplex) technology, tunable lasers, filters and tunable receivers it is possible to combine 4 different wavelengths on a single medium. Technique of TWDM (Time and Wavelength Division Multiplex) is created combining TDM and WDM. NG-PON2 was implemented, WDM was used only for backward compatibility of a given standard with older standards. The main contribution of this paper is an implementation of NG-PON2 communication into OPNET Modeler tool. We also demonstrate a static and dynamic bandwidth allocation. Furthermore, our model proves a novel dynamic bandwidth allocation with modification of MaxMin fair algorithm in which a free grant size is spread to heavily loaded ONU (Optical Network Unit). At this time, several implementations for EPON passive optical network are available in OPNET [4], [5], [6]. However, communication in networks defined by the ITU take place in a different way. There is no high-level protocol as in the case of EPON technology. The associated functional layer is defined as a transmission convergence layer. Defined data units are many times larger than the Ethernet frames. The question of dynamic bandwidth allocation is widely discussed [7], [8], however the area of EPON network dominated initially. This changed with the arrival of XG-PON networks [9], [10]. Our main goal was to implement static bandwidth allocation (although it is not being used in reality), which could be further modified for dynamic models. Utilizing the Min-Max Fair algorithm allows us to take an advantage of free bandwidth for heavyload ONU [11], [12]. Our modifications will also allow the heavy-loaded ONU to benefit from the free grant size. For example, during night time it is possible to use all the free slots for other ONUs. Our simulations clearly demonstrate the basic communication functionality of the NG-PON2 network implanted in OPNET Modeler and provide relevant data for simulation of the dynamic bandwidth allocation algorithm. This implementation also allows for future expansion with additional algorithms for dynamic bandwidth allocation.

The rest of this paper is structured as follows. In the next section an overview of related works is mentioned. 
Section III gives an overview about a grant allocation in NGPON2. Section III-A describes Max-Min fair DBA algorithm. Section IV introduces an example of grant allocation in NGPON2. Section V introduces our implementation of NG-PON2 in OPNET Modeler and describes scenarios and results. Finally, Section VI concludes the paper.

\section{RELATED WORKS}

In recent years, many articles dealing with transmission convergence layer of PONs have been published.

For example, article [13] provides an overview of the activation process for ONU units with real measurements and simulation results. Unfortunately, we dealt with a GPON network which is of older standard in comparison with NGPON2. On the other hand, there are some pilot project with deployment of NG-PON2 [14], [15]. We also dealt with a data analysis in Gigabit passive optical network in [16]. These data are encapsulated into GEM (GPON Encapsulation Method) frames, a GEM frame uses transmission convergence services. Furthermore, a data analysis is not possible to do in real time because a special tool is necessary to be used, in our case it was GPONtracer. GPONtracer contains FPGA (Field Programmable Gate Array) to store a transmitted data from ODN. The further article presented in [17] dealt with transmission converge layer of PON. We focused on equalization delay for each ONU and influence of the refractive index on timing in $\mathrm{XG}-\mathrm{PON}$. Note that XG-PON standard is the first specification of PONs for Next Generation Networks, it is also called as Stage 1 which has to keep a compatibility with previous PON standards.

Nowadays, only tutorials and basic definitions of transmission convergence layer of NG-PON2 are taken into account [18], [19], [20]. All articles introduce only basic information based on standard ITU-T G.989.3 because as mentioned previously, there is no real network for testing. The other important issue in NG-PON2 is a energy management and power consumption because ONUs are active for all-time [21]. If there is a way how to save energy of ONU then all ISPs will welcome it. On the other hand, the energy management process will need to change firmware software which can be difficult because all vendors do not allow it for ISPs. OPNET Modeler does not include an implementation of PONs or an implementation is not public. On the other hand, you are able to use NS-3 (Network Simulator) with XG-PON package for simulation of XG-PON. There are some article which present model of EPON (Ethernet PON) [22], [23], and [24]. More precisely, articles deal with EPON standard so we cannot adopt a model or process models. EPON standard is based on Ethernet frame but NG-PON2 uses a proprietary solution. That is the why, we started with simple model for upstream direction.

\section{Grant Allocation And DBA Algorithm}

To maximize usage of the bandwidth per wavelength in the upstream direction, a proper grant allocation mechanism need to be used. In PONs two types of grant allocation algorithms are used nowadays:
1) Static grant allocation - basic mechanism using predefined parameters. This mechanism is not able to react to dynamic changes in a network.

2) Dynamic grant allocation - advanced mechanism, which is able to react to changes in network using two types of methods to find out the actual state of ONUs. There are the following types of DBA methods [25], [26]:

- Status reporting (SR) - OLT (Optical Line Terminal) unit uses messages to query ONUs for state of their buffers. Buffer size of ONU equals $\approx 1 \mathrm{MB}$ [27], [28]. After receiving replies from ONUs, the OLT unit is able to compute the best grant allocation for every ONU for actual state of the network.

- Traffic monitoring (TM) - in this method the OLT unit checks frames sent by other ONUs to find out if a given ONU is sending relevant frames or idle frames (empty buffers). After acquiring necessary informations, the OLT can change the allocations accordingly.

\section{A. Max-min fair DBA Algorithm}

Max-min fair was introduced for GPON standard networks but after some adjustments it is possible to use this algorithm also in XG-PON (NG-PON) networks. To determine the actual state of the network it is possible to use both SR or TM methods. Complexity of the algorithm is low (polynomial). The Algorithm has some rules about given allocations:

- Allocations cannot be bigger than buffer used for the corresponding ONU.

- Unsaturated ONUs obtain same amount of the remaining bandwidth

Given the max-min fair algorithm, each allocation for ONU $n$ during frame $t$ with grant size $W_{t}^{n}$ is calculated from the following steps [30]:

1) Initialization: Sort ONU demands in an increasing order in set $S$. Set grant size $W_{t}^{n}$ of ONUs to zero.

2) Calculate available bandwidth:

$$
B_{L}=\frac{B W-\sum_{k=1}^{m} W_{t}^{k}}{|S|},
$$

where $B W$ is the total available bandwidth in the upstream direction (frame - (overhead + guard times) $), m$ is the total number of ONUs, and $|S|$ is the number of ONUs in $S$.

3) Update grant size $W_{t}^{n}$ of all ONUs with:

$$
W_{t}^{k}=\min \left\{R_{t}^{k}, W_{t}^{k}+B_{L}\right\},
$$

where $R_{t}$ represents a request for $K_{t h}$ ONU.

4) Remove all fully saturated $\left(W_{t}^{k}=R_{t}^{k}\right)$ ONUs from $\mathrm{S}$.

5) If $|S| \geq 0$ than repeat from 2. End if $|S|=0$.

\section{EXAMPLE OF GRANT ALLOCATION IN NG-PON2}

For description of grant allocation, it is necessary to understand the communication between an OLT unit and ONU units. Basic situation consisting of minimum units in ODN (Optical Distribution Network) is shown in Fig. 1. 


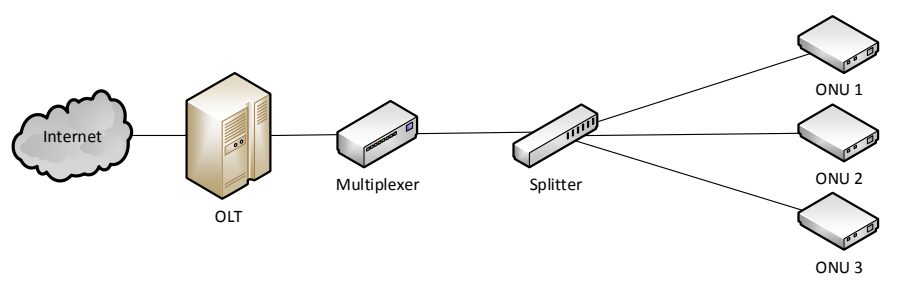

Fig. 1. Basic topology.

Data in NG-PON2 are transmitted in XGEM (XG-PON Encapsulation Method) frames that are shown in Fig. 2 where the most important field denoting addressee of XGEM frame is called XGEM Port-ID. XGEM Port-IDs are assigned during registration process. Remaining fields of XGEM frame:

- PLI (Payload Length Indication) - field indicating the size of payload.

- Key Index - indicates if encryption for payload is used.

- Options - for future use, set to 0x00000, ignored by receiver.

- LF (Last Fragment) - field indicating if SDU (Service Data Unit) was fragmented.

- HEC (Hybrid Error Correction) - used for detection and correction of transmission errors.

Another important part of the downstream physical frame is the overhead consisting of multiple fields.

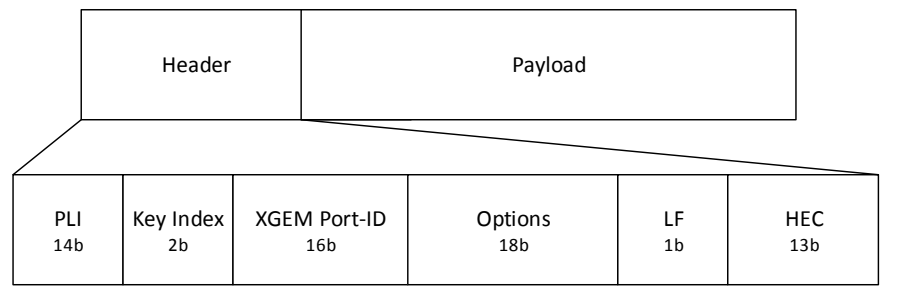

Fig. 2. NG-PON2 XGEM frame.

For our description, the overhead field called BWmap is important as shown in Fig. 3.

BWmap is formed out of many fields. The most important fields are:

- Alloc-ID - 14 bit identifier of addressee in the upstream direction.

- Start Time - field of BWmap indicating interval when the first byte should be transmitted (physical upstream frame is divided to 9720 intervals, value between 0 and 9719 is expected).

- Grant Size - field indicating the size of data part and DBRu (Dynamic Bandwidth Report in upstream) in allocation. Size of the field depends on a given ONU transmission rate.

In our case, only one XGEM frame (from OLT to ONU) and one allocation (for ONU 1) are monitored and described. The attributes of described allocation are: Alloc-ID $=100$, StartTime $=0$, GrantSize $=200$, and the attribute of XGEM frame is XGEM Port-ID $=100$.

Description of communication defining a grant allocation starts at an OLT unit (OLT CT - Optical Line Terminal Channel Termination), when a downstream physical frame is sent

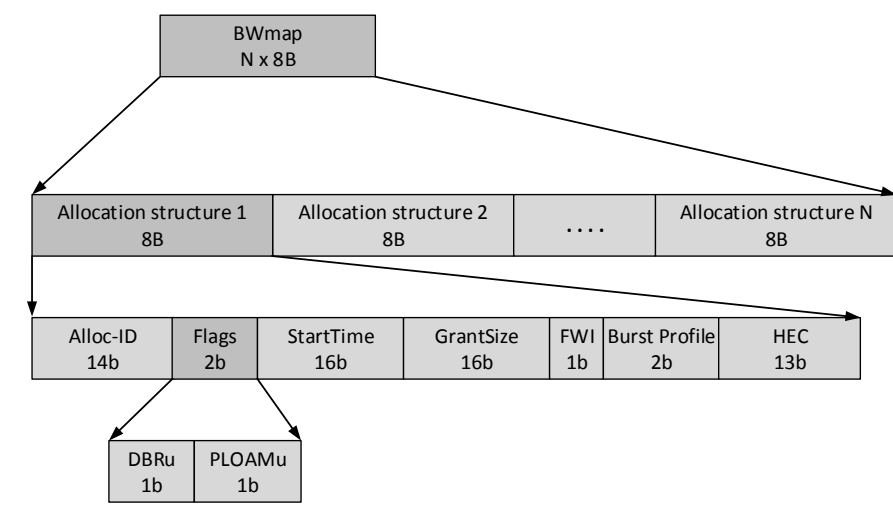

Fig. 3. NG-PON2 BWmap.

(including described downstream XGEM frame) on defined wavelength. Then, the frame is multiplexed in a multiplexer with other transmissions on different wavelengths. The process and node model of OLT are shown in Fig. 4 and 5. Node model OLT source processor generates queries for ONUs to report their buffers. Processor packet_check_set_parameters checks incoming reports and set corresponding parameters, that are used by source processor in next periods of grant allocations. Node model ONU processor proc processes incoming data, if data are not for the unit, frame is transferred to sink, if data are for the mentioned ONU, data are processed, parameters of $\mathrm{ONU}$ are set correspondingly and frame is sent to sink_my_packet. Proc also generates traffic that is transferred to the OLT in corresponding time. Next node in our topology (also in real networks) is a splitter, which is passive and does not process any data. This node only divides incoming signals on different wavelengths to its output ports. In our implementation, a splitter is not full passive element (it processes data) because we are not able to implement a completely passive element in OPNET Modeler (see Fig. 6). Last nodes in an ODN are ONU units (sometimes also called ONT). ONU units receives all transmissions on defined wavelength, all other transmissions are not detected, although the ONU units can be set to different wavelengths by the OLT, this is called tuning. Tuning process is a part of an activation process for each ONU. ONU is able to select different wavelength from OLT CT [29]. In our case, the ONU 1 receives all traffic but it processes only valid allocation with Alloc-ID 100 and the XGEM frame with XGEM Port-ID 100. Information from filtered allocation structures are processed, which means the ONU unit will start its transmission (data part) at the start of the upstream physical frame and it will transmit $3200 \mathrm{~B}(200 \times 16 \mathrm{~B})$ of data (including DBRu) with transmission rate $9.95328 \mathrm{Gbit} / \mathrm{s}$. For the upstream transmission, defined wavelength is used. In the upstream direction, transmissions with different wavelengths are combined together in the splitter. In the demultiplexer, incoming transmissions are filtered based on the wavelength used for the corresponding OLT CT. The OLT checks if data were transmitted in correct time interval, if not, it synchronizes the ONU unit. Described principle is the same for all ONUs and wavelengths. 


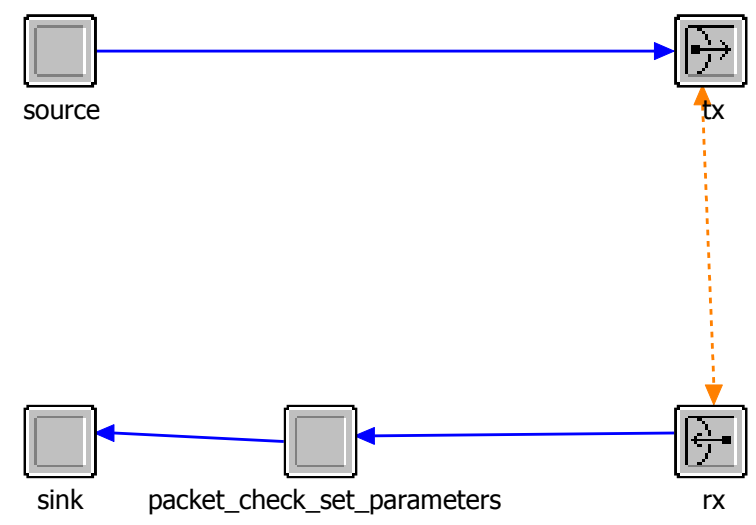

Fig. 4. OLT node model.

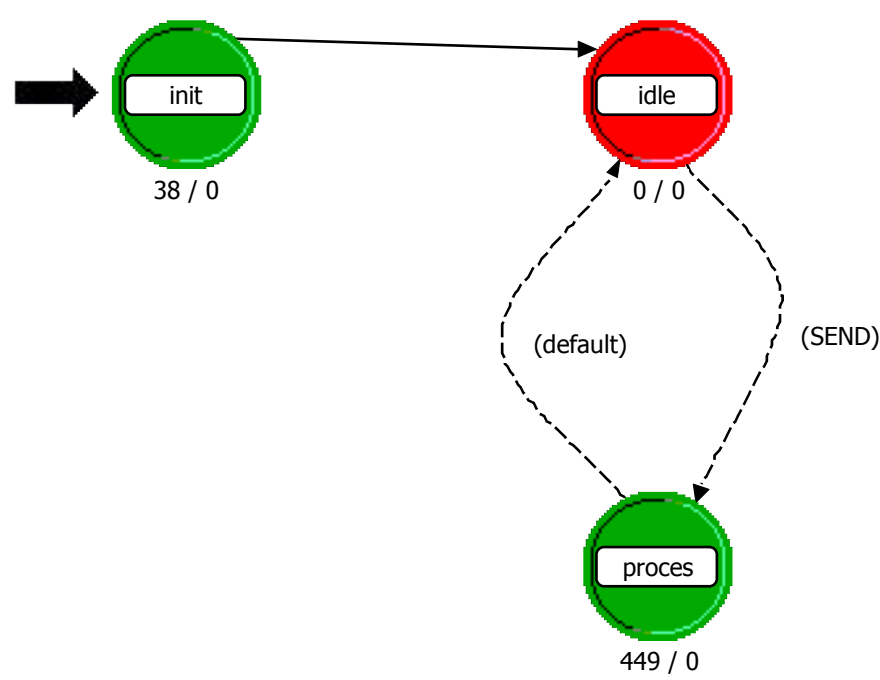

Fig. 5. Process model of OLT.

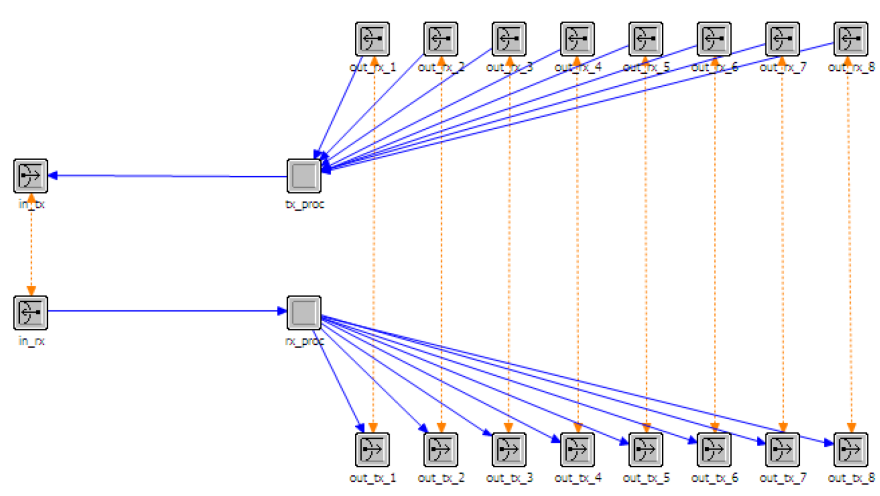

Fig. 6. Designed splitter with split ratio of 1:8.

\section{ImPlEMENTATION AND SimUlations RESUlts}

For implementation of NG-PON2 standard and simulations of a grant allocation OPNET Modeler was chosen. In OPNET Modeler known also as Riverbed Modeler, no package for optical units or transmissions is available. For simulations, all optical units, processes and fiber had to be designed in different layers of the OPNET Modeler.

For simulations, all necessary data units (BWmap, XGEM frame), all nodes and links were designed. To simulate static and dynamic grant allocation without changing the topology, OLT CT and ONU units contain the most important attributes of both types of allocation. The OLT CT units can be set with the following attributes:

- Basic ONU-ID - attribute defining start number (ONUID) of a node on the according wavelength. ONU-IDs are identical to Alloc-IDs.

- Guard time - defines guard time interval between two bursts.

- Mode - this attribute represents a switch between static and dynamic allocation (value 0 means static allocation and value 1 means dynamic allocation of this OLT CT).

- Modification - by this attribute, modification of Max-Min fair DBA can be turned on (discussed later).

- ONU 1-8 - activation of according ONU is set here, defined logically.

- Offset - advance of US and DS physical frame can be set by this attribute.

- Period - attribute defining US and DS physical frame size.

- Start time - defines time when the OLT turns to an active state.

Accordingly, OLT and all activated ONUs must be set with these parameters:

- ONU-ID - defines ONU-ID of a given node.

- Seed - initial number of a random generator. Different value needs to be set in every ONU (for different requests of bandwidth in one period).

- Frame size - attribute that allows to change time of the physical frames (responds to Period of the OLT CT). Every ONU unit can request up to $40 \%$ of physical upstream frame.

All mentioned parameters are used by the process model of ONU unit shown in Fig. 7. ONU nodes model shows Fig. 8.

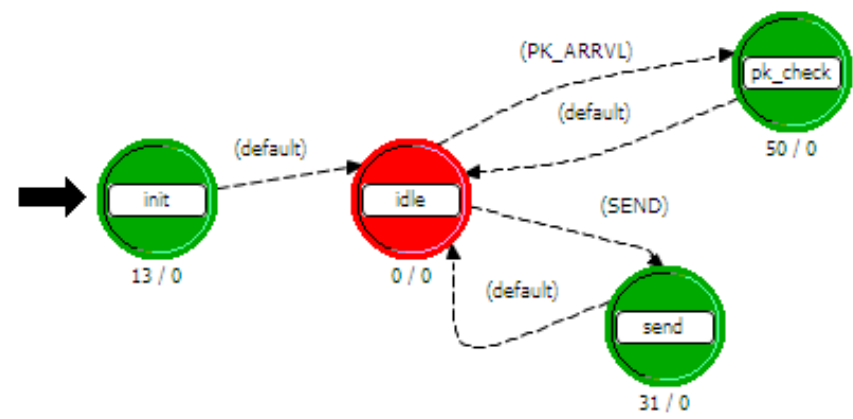

Fig. 7. Process model of ONU unit.

\section{A. Scenario 1}

For simulations, a basic topology shown in Fig. 9 was created.

Scenario 1 simulates a static grant allocation (one wavelength), when time of the physical upstream frame is divided among ONUs equally. Results seen in Fig. 10 describe the 


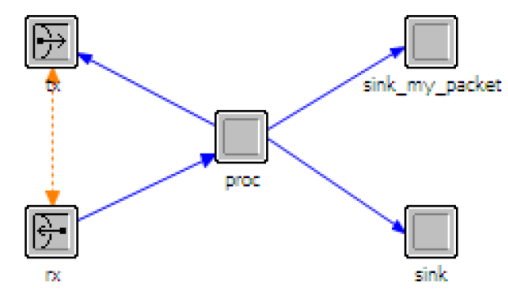

Fig. 8. Nodes model of ONU implementation.

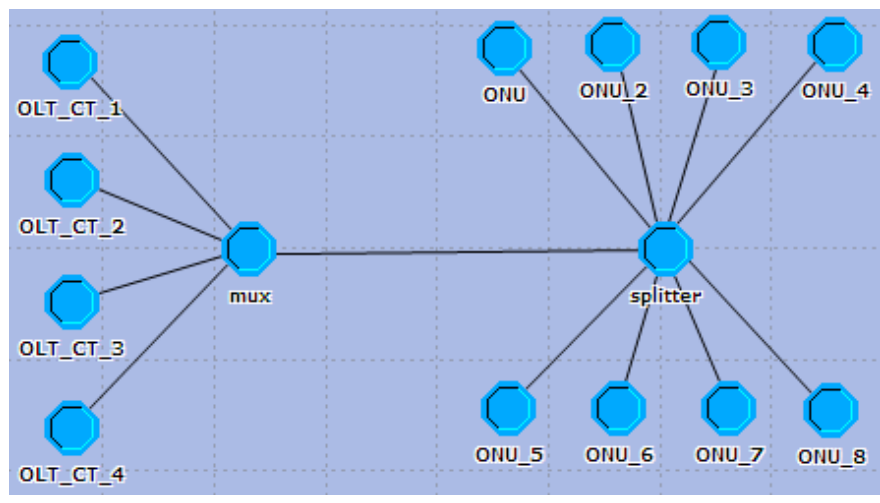

Fig. 9. Designed topology in OPNET Modeler.

static allocation of 8 ONUs in two physical upstream frames and confirm our assumptions.

Our assumption was that the physical US frame would be evenly distributed among all ONU units. According to the displayed results of the simulation, the associated frame was extended approx. $1000 \times$ (therefore, its size is $125 \mathrm{~ms}$ ). Due to the size of the guard interval ( $3 \mathrm{~ms}$ ) between bursts of ONU units, the results are displayed in a $3 \mathrm{D}$ chart, which is clearer as opposed to the 2D chart, which would merge the transmission of all displayed ONU units.

\section{B. Scenario 2}

This scenario shows results of the static grant allocation while multiple OLT CTs are active. In Fig. 11 it is shown that five ONUs transmit using $\lambda_{1}$ while three ONUs transmit with $\lambda_{2}$.

In comparison with ONUs 6-8 (simulation units), which are less active on the respective $\lambda_{2}$, ONUs $1-5$ transmitting on $\lambda_{1}$ do receive smaller grants, thus they are statically provided with allocated larger grants. Transmission of multiple signals to a single medium is possible thanks to WDM used in NGPON networks. By dividing the units into larger/smaller groups on the respective $\lambda$, in some cases, a certain bandwidth difference per ONU can be achieved (a smaller number of units with larger requirements are assigned to a given $\lambda_{2}$ as opposed to a group of multiple units that are active on $\lambda$ and do not need high bandwidth). Nevertheless, since not using the NG-PON networks is not efficient (both financially and functionally), there is no advantage of using the static allocation of grants (even if adjusted as described above).

\section{Scenario 3}

In this scenario a dynamic allocations with Max-Min fair algorithm was simulated.

Commonly, a physical upstream frame is divided accordingly to ONUs' requests (described in section III). If requests from ONUs are bigger than the physical upstream frame, the results of the grant allocation are similar to the results in Fig. 10.

The results of this scenario are shown in Fig. 12 where nonstandard situation is simulated. Due to low requests of ONUs connected with OLT CT, physical upstream frame in the third period is not utilized completely. This situation can occur at night, during power outages etc.

The first grant allocation period is governed by a static allocation procedure (the initial simulation data does not contain any information about loading of the ONU queues at the start of the simulation). In the second period, there is a slight change compared to the first period (static allocation). There is a reduction in requirements of both ONU 2 and ONU 4 , which leads to an increase in the allocations for ONU 1 and ONU 3 that reported high memory usage. The change in the requirements of a particular ONU in the third period is clearly visible when there is a situation in which the US frame is not fully utilized and approx. 15-20\% of the frames capacity is not used. This loss is caused by the properties of the MaxMin Fair algorithm: OLT can only provide ONU units with the amount of transmission time that is actually requested by these units (see Chapter IV). The fourth period is very similar to the first two periods, as the units sent a larger allocation request (in total) than the size of the US frame.

\section{Scenario 4}

Imperfection of scenario 3 is rectified by our modification of Max-Min Fair algorithm. This modification redistributes remaining part of the physical upstream frame equally to all ONUs. The results are shown in Fig. 13. This modification breaks the rule that says: Allocations cannot be bigger than used buffer of the corresponding ONU. Although this rule was broken, all ONUs received the allocation that was requested. Such excessive allocation assigned dynamically could be utilized in situations with enabled fast changes of the requested bandwidth.

The results of our simulation clearly show that the allocations for all units increase by a fraction of the unused part of the US frame. Any important change in the allocations is not available until the fourth Allocation Period. Since the time during which a DBA should be able to modify the distribution of allocations to $2 \mathrm{~ms}$ (based on target requests), the allocated grant may not meet the allocation-specific requirements of the ONU unit from the previous period (in reality, the period is approx. $125 \mu \mathrm{s}$, perhaps few more periods are necessary to react accordingly). For this reason, it can be assumed that in our simulation there was an increase of the allocationspecific requirements for ONUs 3-5, however the algorithm did not react to this change successfully. Nevertheless, thanks to the modification of the algorithm these units received larger allocations than they would have obtained without this 


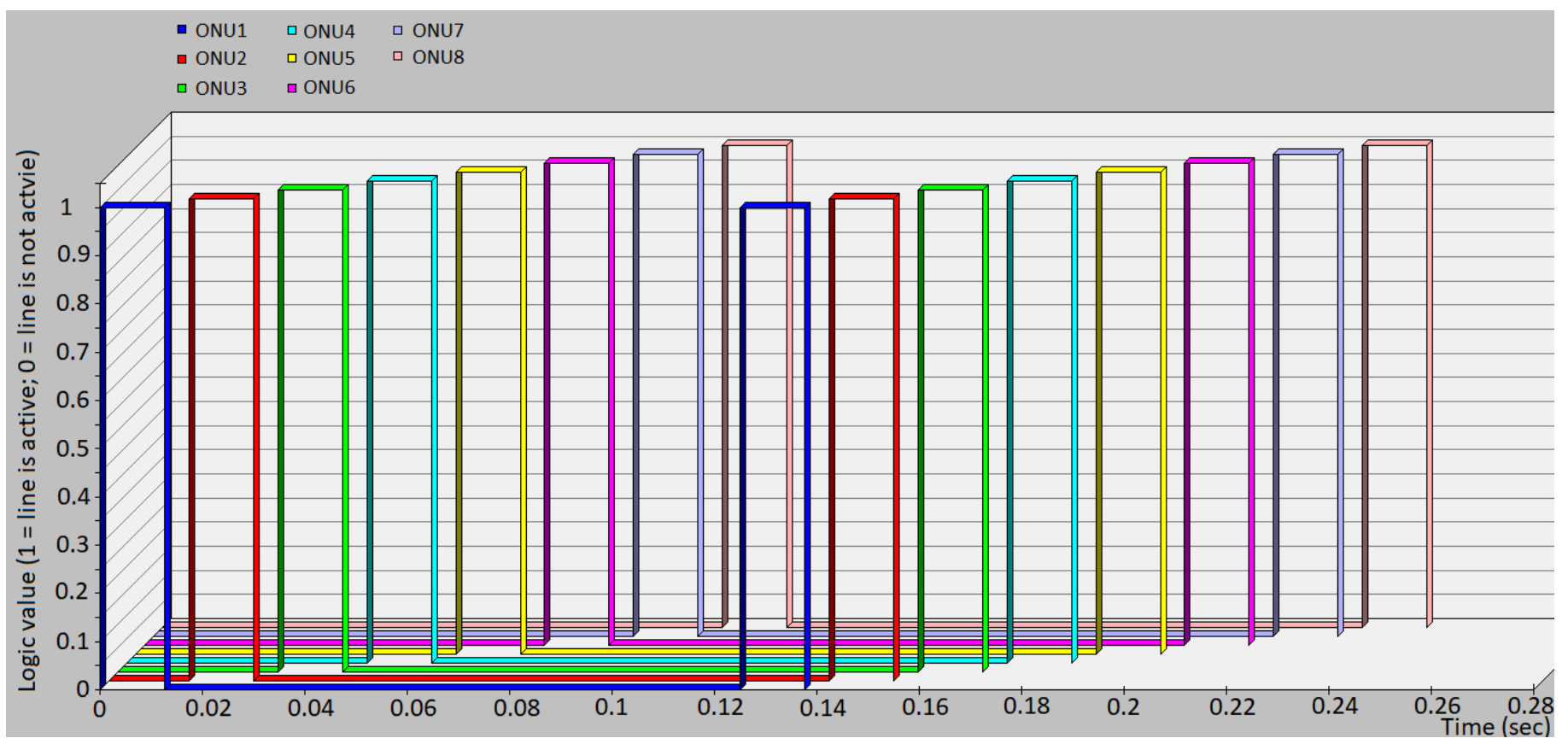

Fig. 10. 3D results of static grant allocation in Scenario 1.

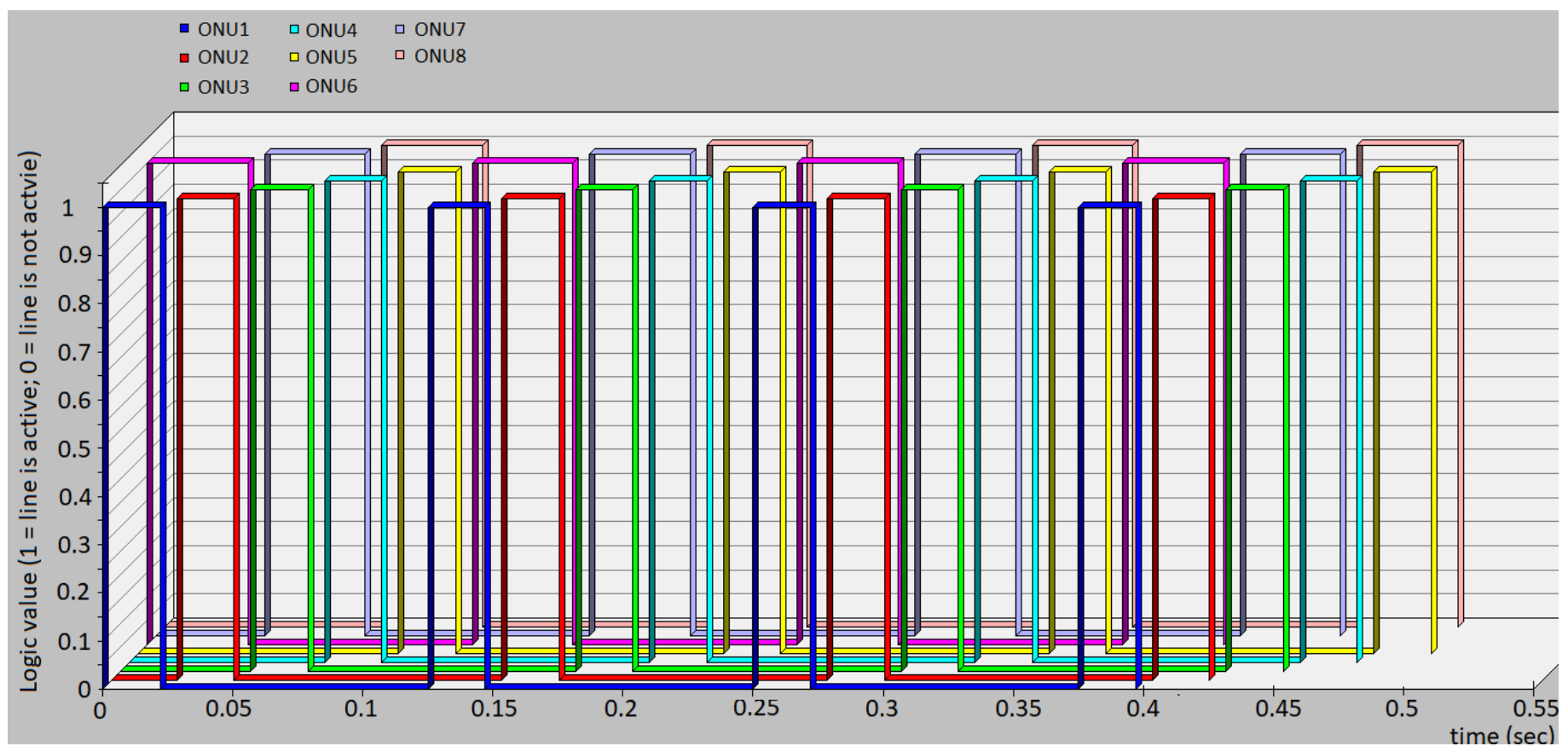

Fig. 11. 3D results of static grant allocation in Scenario 2 (two OLT CT are activated).

modification and they were able to send more data in their allocation during this particular period. This implies that these units required smaller allocations during the next period. In consequence, ONU 1 and ONU 2 did receive larger grants which enabled them to send more data and also to empty their queues more efficiently, which subsequently affected the next period that is no longer displayed. The simulation results are not supported by real data, but they do reflect the theoretical behaviour of the network in this situation.

\section{E. Scenario 5}

This scenario compares time requirements of simulations mentioned earlier. In this scenario, 5 ONUs were activated for each OLT CT. We assumed an increase of the time needed for simulation of previously mentioned scenarios together with the increase of complexity. Our assumption was confirmed, values are shown in Tab. I. 


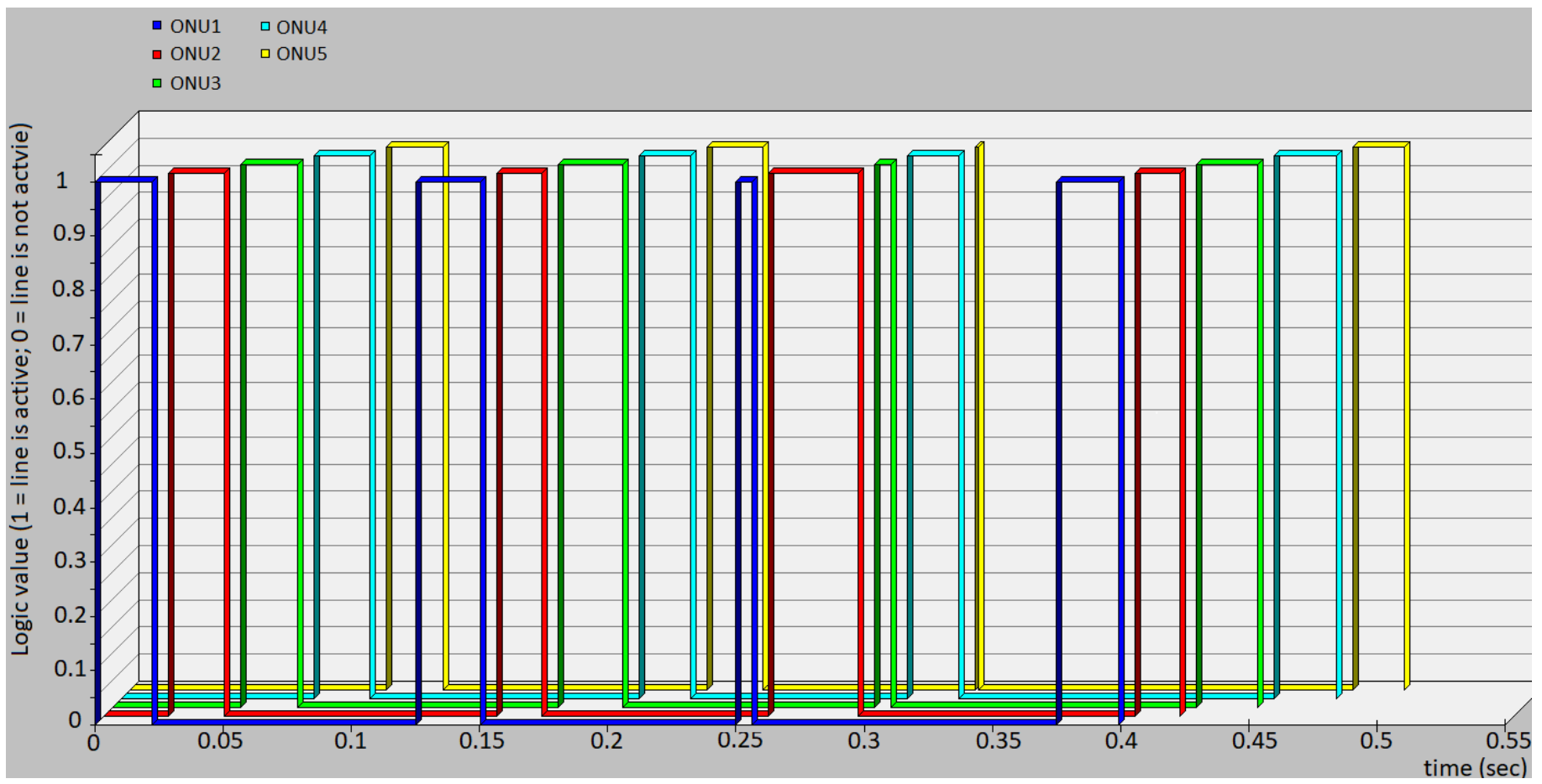

Fig. 12. 3D results of dynamic grant allocation.

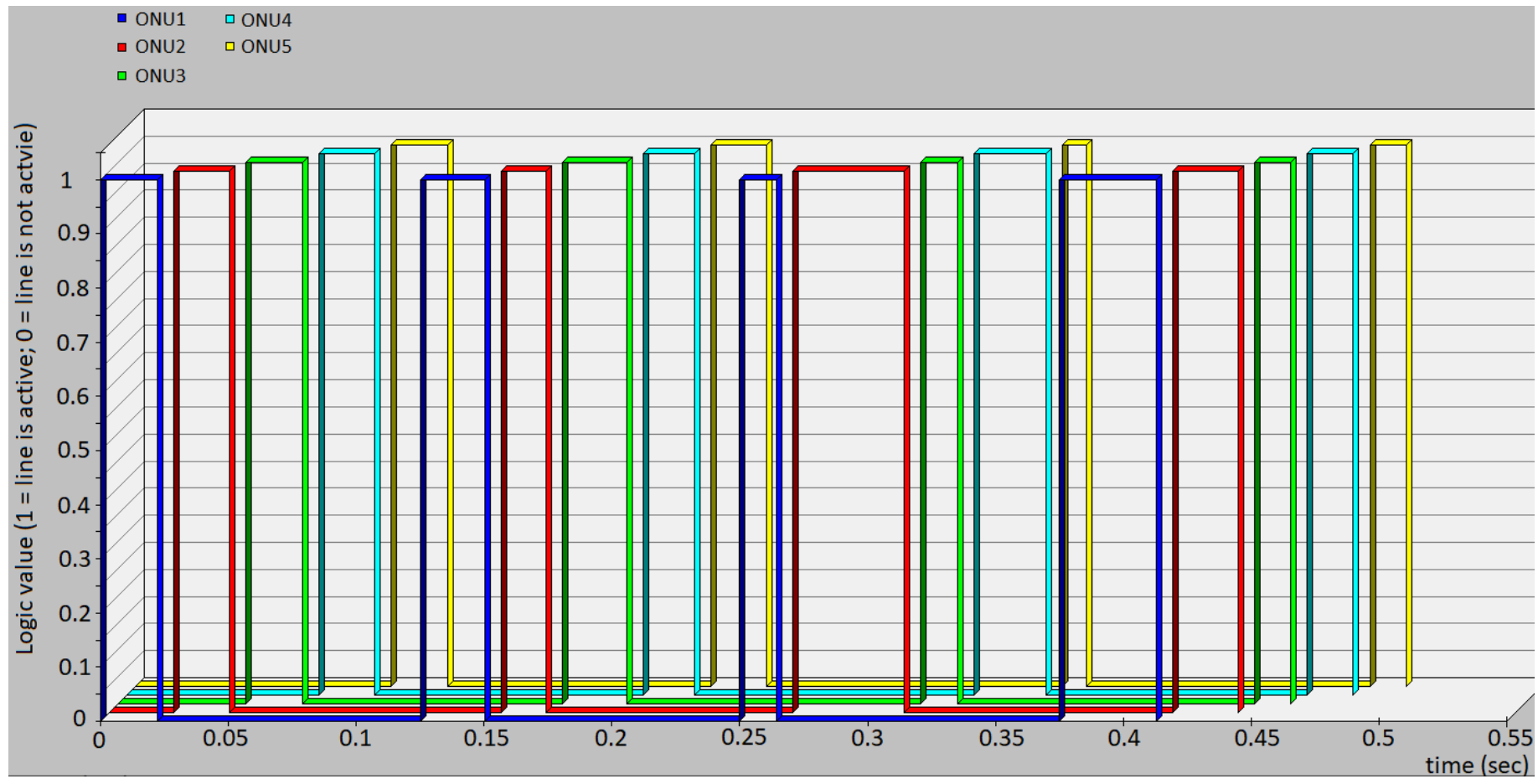

Fig. 13. 3D results of dynamic allocation with modification.

\section{CONCLUSION}

NG-PON2 will probably be a promising solution for passive optical networks in 3-5 years because current prices of OLT and ONUs are too high. We have implemented a light version of NG-PON2 communication in the OPNET Modeler tool. OPNET Modeler is a powerful simulation tools for IT technologies simulations.
Unfortunately, it does not contain a PON implementation. Proposed results show that our implementation splits up the grant size between each ONU. For dynamic bandwidth allocation, Max-Min fair algorithm was used. However, an unused grant should not be used for other ONUs but it can help to decrease a buffer-size at the ONU. Note that, our simulation model contained only 8 ONUs. Real networks provide 
TABLE I

VALUES OBTAINED IN SCENARIO 5.

\begin{tabular}{|c|c|c|c|}
\hline Seconds simulated & $\mathbf{1 0}$ & $\mathbf{6 0}$ & $\mathbf{1 8 0}$ \\
\hline Time needed to simulate static allocation [s] & 6,8 & 40 & 120 \\
\hline Time needed to simulate dynamic allocation [s] & 10 & 58 & 170 \\
\hline Time needed for simulation with modification [s] & 10 & 63 & 195 \\
\hline
\end{tabular}

a connectivity for up to 256 ONUs per PON port. However, a simulation of such split ratios would be time inefficient. Furthermore, lower split ratio provided well-arranged results, which prove the fair grant allocation.

The future research will continue with implementation of another DBA algorithm (such as GIANT) into our simulation model and testing algorithm on real optical networks. Furthermore, we would like to implement own implementaion of the OLT transponder.

\section{ACKNOWLEDGEMENT}

Presented research has been supported by project of the Ministry of Interior under grant no. VI20172019072 registration and the National Sustainability Program under grant LO1401.

\section{REFERENCES}

[1] G.984.2 : Gigabit-capable Passive Optical Networks (G-PON): Physica Media Dependent (PMD) layer specification, 2003. [Online]. Available: http://www.itu.int/rec/T-REC-G.984.2/en.

[2] G.987.2 : 10-Gigabit-capable passive optical networks (XG-PON): Physical media dependent (PMD) layer specification, International Telecommunication Union, 2016. [Online]. Available: https://www.itu.int/rec/TREC-G.987.2/en.

[3] G.989.2 : 40-Gigabit-capable passive optical networks 2 (NGPON2):: Physical media dependent (PMD) layer specification-, International Telecommunication Union, 2014. [Online]. Available: https://www.itu.int/rec/T-REC-G.989.2/en.

[4] A. F. Y. Mohammed, S. H. Shah Newaz, and J. K. Choi, Understanding TCP throughput performance in TDM-PONs with sleep mode, in 2014 12th International Conference on Optical Internet 2014 (COIN), 2014, pp. 1-2, DOI: 10.1109/COIN.2014.6950622.

[5] A. F. Y. Mohammed, S. H. S. Newaz, and J. K. Choi, Modeling and simulation of EPON with sleep mode enabled using OPNET, in 2014 International Conference on Information and Communication Technology Convergence (ICTC), 2014, pp. 16-21, DOI: 10.1109/ICTC.2014.6983076.

[6] A. F. Yousif Mohammed, S. H. S. Newaz, M. R. Uddin, G. M. Lee, and J. K. Choi, Early Wake-Up Decision Algorithm for ONUs in TDM-PONs With Sleep Mode, Journal of Optical Communications and Networking, vol. 8, no. 5, pp. 308-319, 2016, DOI: 10.1364/JOCN.8.000308.

[7] M. M. Rahman and M. Hossen, Modified hybrid slot-size/rate DBA algorithm for improving quality of services of high priority data packets of PON system, in 2016 5th International Conference on Informatics, Electronics and Vision (ICIEV), 2016, pp. 391-395, DOI: 10.1109/ICIEV.2016.7760032.

[8] Li Li, Xin Shouting, and Duan De-Gong, Research of DBA schemes and QoS in PON system, in 2016 2nd IEEE International Conference on Computer and Communications (ICCC), 2016, pp. 2148-2153, DOI: 10.1109/CompComm.2016.7925080.

[9] P. Sarigiannidis, M. Louta, G. Papadimitriou, I. Moscholios, A. Boucouvalas, and D. Kleftouris, Alleviating the high propagation delays in FiWi networks: a prediction-based DBA scheme for 10G-EPON-WiMAX systems, in 2015 International Workshop on Fiber Optics in Access Network (FOAN), 2015, pp. 45-50, DOI: 10.1109/FOAN.2015.7320478.

[10] C. A. Kyriakopoulos and G. I. Papadimitriou, Bandwidth efficiency in the next generation access architecture XG-PON, in 2016 Eighth International Conference on Ubiquitous and Future Networks (ICUFN), 2016, pp. 833-838, DOI: 10.1109/ICUFN.2016.7537154.
[11] J. A. Arokkiam, K. N. Brown, and C. J. Sreenan, Optimised QoS-Aware DBA Mechanisms in XG-PON for Upstream Traffic in LTE Backhaul, in 2016 IEEE 4th International Conference on Future Internet of Things and Cloud Workshops (FiCloudW), 2016, pp. 361-368, DOI: 10.1109/WFiCloud.2016.79.

[12] N. Merayo, P. Pavon-Marino, J. C. Aguado, R. J. Durn, F. Burrull, and V. Bueno-Delgado, Fair Bandwidth Allocation Algorithm for PONs Based on Network Utility Maximization, Journal of Optical Communications and Networking, vol. 9, no. 1, pp. 75-86, 2017, DOI: 10.1364/JOCN.9.000075.

[13] T. Horvath, P. Munster, M. Jurcik, L. Koci, and M. Filka, Timing measurement and simulation of the activation process in gigabit passive optical networks, Optica Applicata, vol. 45, no. 4, p. 459-471, 2015, DOI: $10.5277 / \mathrm{oa} 150403$.

[14] Verizon to begin NG-PON2 deployment in 1Q18, TeleGeography, 2018 [Online]. Available: https://bit.ly/2BOVZum. [Accessed: 27-Aug.-2018].

[15] A. Diana, Verizon, Calix Deploy Commercial NG-PON2, BROADBAND WORLD NEWS, 2018. [Online]. Available: https://bit.ly/2MRO2sW. [Accessed: 27-Aug.-2018].

[16] T. Horvath and L. Dubravec, Deep data analysis in gigabit passive optical networks, Optica Applicata, vol. 47, no. 1, p. 157-170, 2017, DOI: 10.5277/oa170114.

[17] L. Koci, T. Horvath, P. Munster, M. Jurcik, and M. Filka, Transmission convergence layer in XG-PON, in 2015 38th International Conference on Telecommunications and Signal Processing (TSP), 2015, p. 104-108, DOI: $10.1109 /$ TSP.2015.7296232.

[18] D. Nesset, NG-PON2 Technology and Standards, Journal of Lightwave Technology, vol. 33, no. 5, p. 1136-1143, 2015,DOI: 10.1109/JLT.2015.2389115.

[19] D. A. Khotimsky, NG-PON2 transmission convergence layer: A tutorial, in 2015 European Conference on Optical Communication (ECOC), 2015 p. 1-3,DOI: 10.1109/ECOC.2015.7341932.

[20] D. A. Khotimsky, NG-PON2 Transmission Convergence Layer: A Tutorial, Journal of Lightwave Technology, vol. 34, no. 5, pp. 1424-1432, Mar. 2016, DOI: 10.1109/JLT.2016.2523343.

[21] R. Wang, P. Bhaumik, H. H. Lee, S. S. Lee, and B. Mukherjee, Energy Management in NG-PON2, in Optical Fiber Communication Conference, 2014, p. Tu3C.4, DOI: 10.1364/OFC.2014.Tu3C.4.

[22] R. Mastrodonato and G. Paltenghi, Analysis of a bandwidth allocation protocol for ethernet passive optical networks (EPONs), in Proceedings of 2005 7th International Conference Transparent Optical Networks, 2005, 2005, pp. 241-244, DOI: 10.1109/ICTON.2005.1505795.

[23] Z. Peng and P. Radcliffe, Modeling and simulation of Ethernet Passive Optical Network (EPON) experiment platform based on OPNET Modeler, in 2011 IEEE 3rd International Conference on Communication Software and Networks, 2011, pp. 99-104, DOI: 10.1109/ICCSN.2011.6013671.

[24] M. Giuntini, J. Morabito, A. Valenti, F. Matera, V. Carrozzo, and S. Di Bartolo, Integration of optical telecommunications and radio access networks to assure quality of service, in 2010 12th International Conference on Transparent Optical Networks, 2010, pp. 1-4, DOI: 10.1109/ICTON.2010.5549109.

[25] P. Sarigiannidis, G. Papadimitriou, P. Nicopolitidis, E. Varvarigos, K Yiannopoulos, HYRA: An efficient hybrid reporting method for XG-PON upstream resource allocation, in 2014 5th International Conference on Optical Communication Systems (OPTICS), 2014, pp. 1-10.

[26] B. Skubic, B. Chen, J. Chen, J. Ahmed, L. Wosinska, Improved scheme for estimating T-CONT bandwidth demand in status reporting DBA for NG-PON, in 2009 Asia Communications and Photonics Conference and Exhibition (ACP), 2009, pp. 1-6.

[27] G. C. Sankaran and K. M. Sivalingam, Report Duration Computation Schemes in Reduced-Buffer Optical Network Units for Passive Optical Networks, Journal of Optical Communications and Networking, vol. 5, no. 11, pp. 1157-1167, 2013, DOI: 10.1364/JOCN.5.001157.

[28] V. Vishnevsky and D. Kozyrev, Eds., Distributed computer and communication networks: 18th International Conference, DCCN 2015, Moscow, Russia, October 19-22. New York, NY: Springer Berlin Heidelberg, 2016

[29] G.989.3 : 40-Gigabit-capable passive optical networks (NG-PON2): Transmission convergence layer specification, International Telecommunication Union, 2015. [Online]. Available: https://www.itu.int/rec/T-RECG.989.3/en.

[30] I. Gravalos, K. Yiannopoulos, G. Papadimitriou, A. E. Varvarigos, A modified max-min fair dynamic bandwidth allocation algorithm for XGPONs, in 2014 19th European Conference on Networks and Optical Communications, 2014, pp.57-62, DOI: 10.1109/NOC.2014.6996828. 


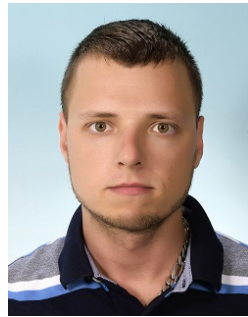

Tomas Horvath (MSc) was born in Havirov, Czech Republic in 1989. He is a PhD student at Brno University of Technology and a researcher at CESNET. He received his MSc and BSc degrees in communications and informatics from Brno University of Technology in 2011 and 2013, respectively. His record shows more than 27 peer reviewed proceedings and journal papers. His current research interests include software-defined optical networking, passive optical networks, and sensing. He is a student member of SPIE, IEEE and OSA.

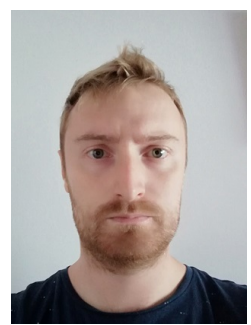

Patrik Cymorek (MSc) was born in Czech Republic in 1992. He received his MSc and BSc degrees in communications and informatics from Brno University of Technology in 2015 and 2017, respectively.

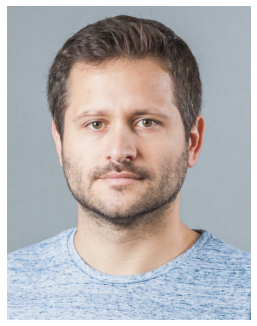

Petr Munster (MSc, Ph.D.) received his MSc degree in communication and informaticsin 2010 from the Brno University of Technology and his PhD degree in 2014. Currently, he is a researcher at CESNET a.l.e. and at Brno University of Technology. His record shows more than 45 peer reviewed proceedings and journal papers in the field of fiberoptic sensing and fiber-optic telecommunications. $\mathrm{He}$ is a member of SPIE, IEEE,OSA, Photonics21, and the Czech and Slovak Society for Photonics.

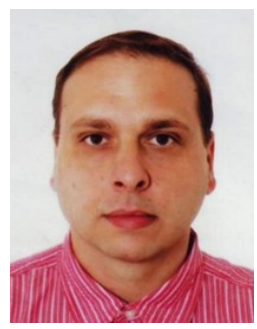

Vaclav Oujezsky (MSc) was born in Brno, Czech Republic. Post graduate student at Brno University of Technology, Department of Telecommunications, Senior Network Engineer at T-Mobile CZ, and currently at IBM CZ. Working actively on projects of security and transport networks at laboratory SIX. His research interests include implementation of evolutionary algorithm, Cisco, Python, VHDL, and converged networks. His topic of dissertation thesis is Converged Networks and Traffic Tomography by Using Evolutionary Algorithms.

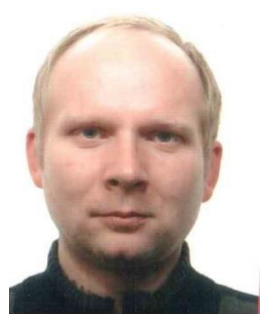

Josef Vojtech (MSc, Ph.D.) is a head of research department of Optical networks at CESNET a.l.e., operator of national research and education einfrastructure of the Czech Republic. He received his MS and BS degrees in computer science and pedagogy from the Czech Technical University in 2001 and 2003, respectively, and his PhD degree in field of optical networking from the Czech Technical University in 2008. His record shows more than 65 peer reviewed proceedings and journal papers. He holds 18 patents (including US and EU) and utility models. He participated in international projects: COMPLETE, FIPPP XIFI, GN4, GN3+, GN3, GN2, PortaOptica Study and SEEFIRE. His current research interests include software defined optical networking and special photonic services as precise time, stable frequency or sensing. He is senior member of SPIE, IEEE, and OSA. In 2007 he received the Research prize of the Czech minister of education. 